\title{
Sur les filaires chez les Équidés et les Bovidés
}

\author{
par Chusaburo SHOHO*
}

II y a déjà une quinzaine d'années que l'étic logie d'une maladie du système nerveux central des animaux domestiques (cheval, mouton et chèvre) était élucidée en Corée par les savants japonais. Elle est causée par des formes jeunes de Setaria digitata (von Linstow, 1906), normalement parasites du péritoine du bœuf domestique en Asie, et qu'on peut trouver en diverses régions de ce continent à la saison où vivent les moustiques vecteurs de cette filaire (Anopheles hyrcans sinensis, Armigeres obturbans et Aedes togoi). Les conséquences que l'on peut tirer de ces observations originales ont été déjà discutées dans nos publications précédentes (Innes et Shoho, 1953, et Shoho, 1954).

Parmi plusieurs problèmes, abordés par nous, le phénomène du complexe neuro-oculaire est une question très intéressante. Au Japon, il y a une différence entre les formes de type nerveux et celles de type oculaire, surtout chez le cheval. Quelques cas sont aussi observés, dont les divers symptômes nerveux sont constatés avant l'apparition du ver dans la chambre antérieure de l'œil. Le phénomène existe aussi, rarement. chez le bouf (Shoho, 1956). Je crois, avec M. Innes, que les filaires accèdent à l'œil en venant de la cavité cérébrale par la fente optique: le phénomène est alors ordinairement observé après celui de la forme nerveuse quelle qu'en soit l'intensité. A ce point de vue, la présence de la filaire dans l'œil du cheval nous suggère la présence du type cérébro-spinal.

La présence de jeunes sétaires dans le globe oculaire est en même temps précieuse pour l'étude des formes jeunes, qui ne sont ordinairement pas visibles dans les tissus de l'hôte, habitat usuel du parasite. Nous avons ainsi eu l'occasion d'observer les jeunes sétaires, que nous savons, maintenant. être assez facilement patho- gènes. Pour lidentification des filiaires oculaires du cheval et du bœuf, on a avantage à se reporter aux travaux publiés au siècle dernier plutôt qu'à considérer les résultats des investigations faites de nos jours. Les ouvrages de Davaine (1877) et Cobbold (1879) avaient envisagé cette étude, non seulement en Europe ou en Amérique, mais aussi en $A$ sie, surtout aux Indes. Le premier commence le chapitre des «Vers (du glabe oculaire) chez les solipèdes » par l'espèce «Filaria papillosa » \{?\} (Synops., n 81\}, mais pour lui, l'identité de tous les vers n'est pas certaine, et " s'ils sont de la même espèce, appartiennent-ils à Filaria papillosa que l'on rencontre dans les autres organes du cheval et de l'âne ? Ces questions ne sont point résolues ». A l'article I, il étudie la filaire d'origine indienne, tandis qu'à l'article II, il traite de ce ver en Europe et en Amérique, car « il ne parait pas se rapporter exactement par ses caractères à la filaire observée aux Indes; en outre, il ne parait pas que les chevaux parasités par la filaire oculaire dans nos pays soient sujets à la faiblesse des lombes; les cas en sont d'ailleurs rares et n'ont été signalés que de loin en loin. La plupart appartiennent à notre siècle. "Au chapitre suivant, relatif aux «Vers (du globe oculaire) du bceuf » ce savant ne dit rien de l'identité des parasites. J'ai rappelé les hypothèses de Davaine seulement parce que je crois que son avis représentait celui des savants de son temps et peut-être d'ailleurs en est-il de même aujourd'hui encore.

Lors de la publication des ouvrages de Davaine et Cobbold, Filaria papillosa (syn. Gordius equi-

(*) Expert du Plan Colombo à Ceylan (décembre 1955iuillet 1957).

Nakayama-Soen Takjrasuska Hyaga-Ken, Japon. 
nus Abildgaard, 1789) comprenait toutes les sétaires, d'origine équine ef bovine. Toutefois, Perroncito en 1882, séparait le parasite du bceuf, qu'il assimilait à Filaria labiato papillare Alessandrini, 1838, de Filaria papillosa. La confusion concernant l'identité des trois sétaires provenant des équidés, bovidés et cervidés est discutée par moi dans un autre travail à paraître prochainement (1958). Ce sont A. Railliet et A. Henry (1911), qui ont signalé pour la première fois, que les jeunes vers rencontrés dans la chambre antérieure de l'œil des chevaux sont des formes jeunes de Setaria labiato-papillosa (Aless., 1838), et non de S. equina (Abildg., 1789) (Syn. F. papillosa). Nous ne savons pas aujourd'hui si ces: auteurs avaient étudié des exemplaires européens ou provenant d'autres parties du monde. Les jeunes sétaires que f'on trouve dans l'œil des chevaux au Japon llchinose et Itagaki, 1945) ou à Ceylan (Nichols and Crawford, 1927) semblent en majorité appartenir à l'espèce Setaria digitata (von Listow). sétaire d'origine bovine, ordinairement trouvée en divers pays d'Asie. Très rarement (seulement 2 sur 111 vers, examinés par Ichinose ef ltagaki) des formes jeunes de $S$. equina sont trouvées avec celles de $S$. digitata mais surtout au printemps, tandis que $S$. digitata est observée à l'automne et en hiver. Schwartz (1927) n'a isolé que des formes jeunes de S. equina dans l'œil des chevaux aux Etats-Unis et au Canada; le phénomène semble en Amériquo moins fréquent qu'en Asie:

Pour nous qui croyons en l'existence du complexe neuro-oculaire, la présence de la nématodose cérébo-spinale, du type nerveux, (« la faiblesse des lombes » de Davaine), est très sugges. tive pour orienter les études et déterminer l'identité de la filaire oculaire dans les diverses parties du monde. Les conditions qui favorisent « la faiblesse des lombes » des poulains et chevaux n'est pas le monopole des Indes. Elles existent aussi au Japon, et sont réunies surtout à la fin de l'été et l'automne, alors que l'encéphalite japonaise est observée ordinairement chez les humains et les chevaux. Ce n'est pas absolument curieux, parce que le virus de cette dernière affection est aussi transmis par des moustiques. Nous avons suffisamment discuté sur la corrélation possible entre ces deux mala. dies d'u. système nerveux central des mammifères
(Innes ef Shoho, 1952). Les troubles identiques étudiés chez des poulains aux Etats-Unis sont connus sous le nom de "Wobbler of foals and yearlings » et il semble que leur histo-pathologie, récemment décrite par Jones et ses collaborateurs (1955), soit parfaitement identique à celle de la nématodose cérébrospinale. Ce fait, discuté par Innes (1957) dans un travail publié en collaboration avec Saunders, convainc ces auteurs de la possibilité d'intervention d'une forme migratrice du nématode. En Europe, il y a plusieurs relations de parésie lombaire, causée par le parasitisme direct d'un nématode, chez les cerfs sauvages (Schwangart, 1918 ef 1940 ; Kerschagl, 1944-1945; Baudet et Verwey, 1951) (Böhm et Supperer, 1955) mais pas chez les animaux domestiques, au moins récemment, tandis que l'observation coricernant Setaria equina (Syn. Gordius equinus) « intra duram et piam matrem » était déjà rapportée par Abildgaard en 17.89. Il est à noter que la distinction de la sátaire bovine n'a été faite par Perroncito qu'un siècle après cette observation.

Eu égard aux faits ci-dessus exposés, l'identification des sétaires jeunes, trouvées dans la chambre antérieure de l'œil des équidés et bovidós, est très intéressante. J'ai eu à ma disposition 3 échantillons provenant de bovins français, 3 exemplaires femelles qui m'ont été envoyées par M. J. Euzéby de Lyon. II s'agissait de trois préparations, montées sur lame et présentant seulement les portions extrêmes (deux antérieures et trois postérieures). L'une des trois est arrivée en mauvais état lla lamelle ayant glissé sur la lamel et les deux autres sans dommages. Les vers ont été lavés dans l'eau physiologique et plongés dans la solution lactophénolique pour les préparer à la microphotographie. Les dessins à la chambre claire et les miciophotographies nous montrent que l'extrémité antérieure est identique à celle de Setaria digitata avec dents latérales (parties latérales du cercles péribuccall de forme triangulaire, et non semi-lunaires, convexes, comme nous pouvons les voir ordinairement sur Setaria labiatopasillosa (cf. microphotographies des sétaires adultes bovines, envoyées de. France et d'Espagne). En ce qui concerne l'extrémité postérieure, trois ont le bouton terminal lisse, dépourvu de pointes mousses : elles sont alors du type digitata. La position relative des deux appen- 
PLANCH! !

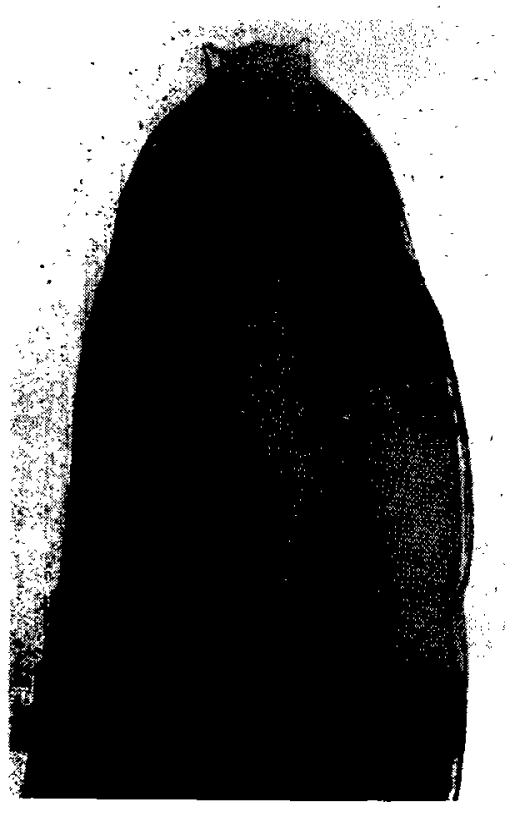

Aa

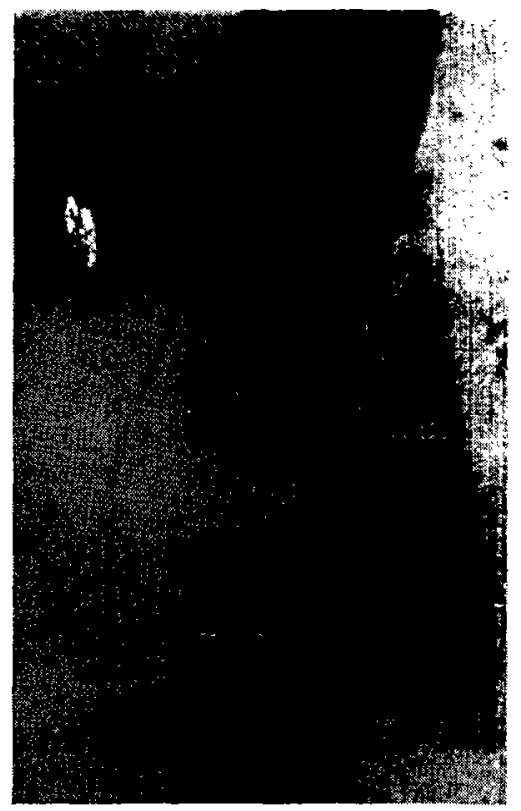

$A b$

Filaire oculaire d'un boûi françuis

Aa : extrémité antérieure.

Ab : extrémité postérieure.

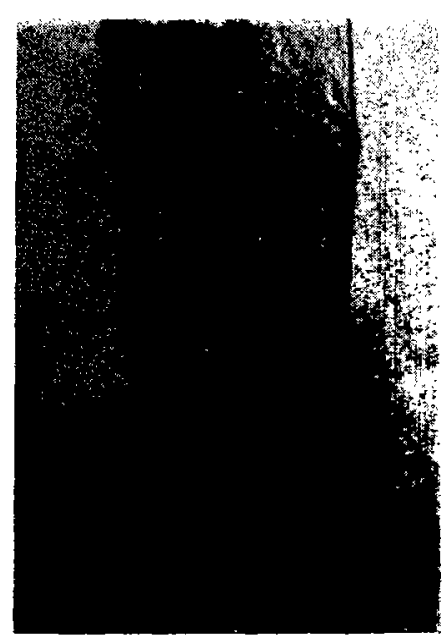

Ba

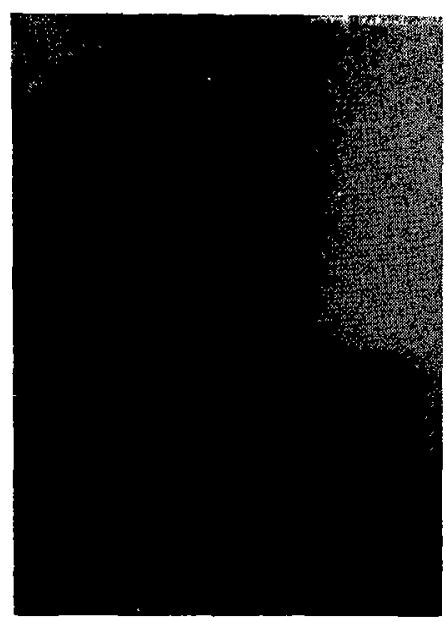

$\mathrm{Bb}$

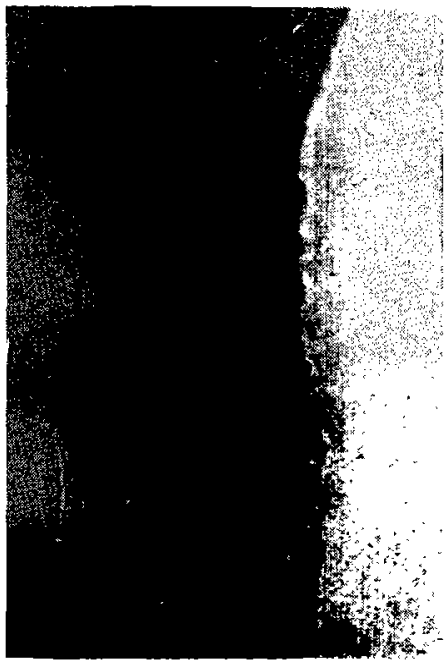

BC

Ba : extrémité antérieure d'une filaire oculaire d'un cheval japonais (la longueur de ce ver est d'environ $35 \mathrm{~mm}$ ). $\mathrm{Bb}$ : extrémité postérieure de la même filaire.

Bc : extrémité postérieure d'un mâle (longueur d'environ $19,5 \mathrm{~mm}$ ). Echantillon provenant de l'œeil d'un cheval japonais. 


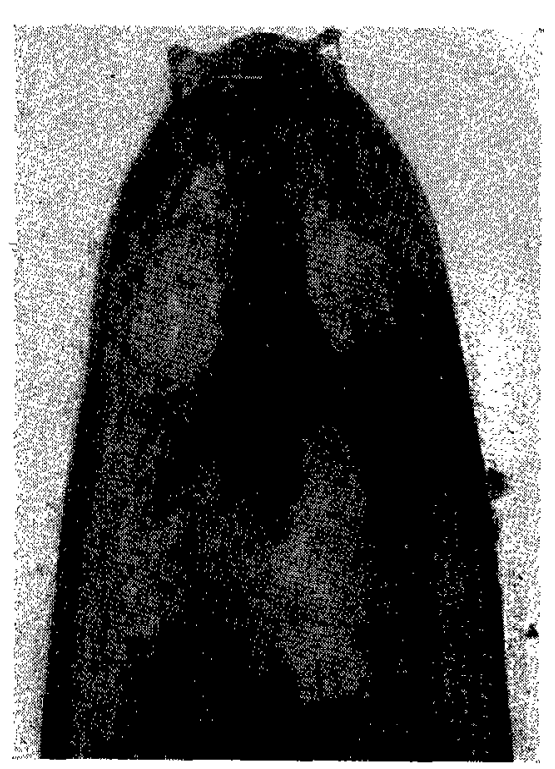

Do

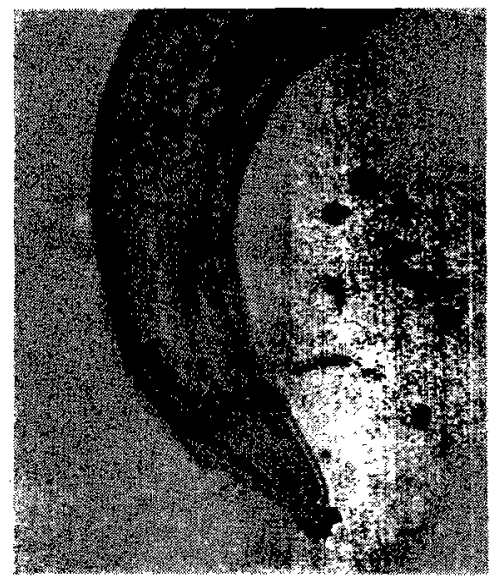

$\mathrm{Db}$

Da : extrémité antérieuce d'une sétaire adulte provenant d'un bœuf français lenvoyée par $M$. le professeur J. Euzéby) S. labiało-papillosa.

$\mathrm{Db}$ : extrémité postérieure de la même sétaire.

N.B. : Photographies $A_{a}$ et $A b$ de la Planche 1 et toutes les photographies de la Planche II sont au même grossissement).

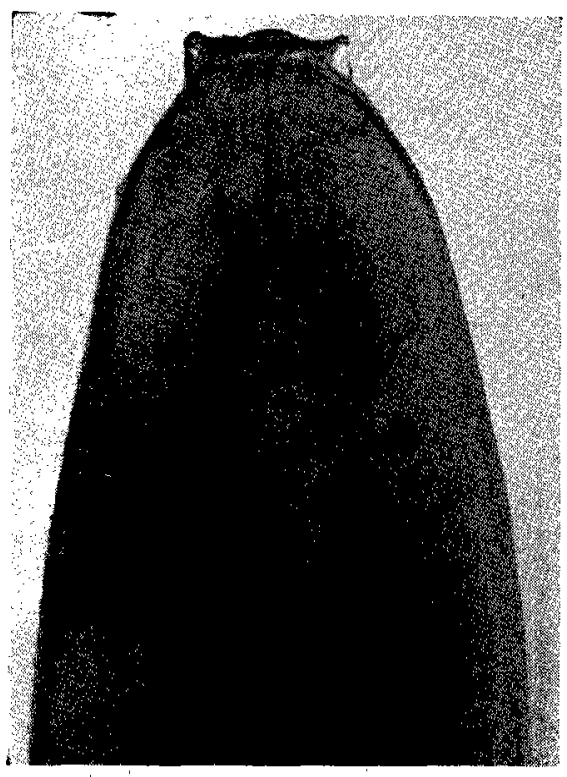

Ea

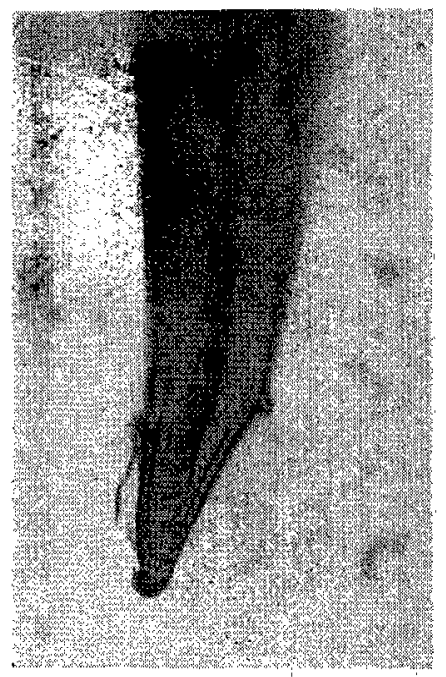

$\mathrm{Eb}$

Ea : extrémité antérieure d'urie sétaire adulte (femelle) provenant d'un bouf espagnol (envoyée par $M$. le professeur C. Granada) S. labiato-papillosa, longueur : envion $85 \mathrm{~mm}$.

Eb : extrémité postérieure de la même sétaire. Remarquer l'extrémité terminale sans petites pointes mousses, comme chez Setaria digitata à Ceylan, mais les dents latérales semblent typiques de S. labiatopapillosa, elles apparaissent avec une fórme semilunaire convexe en vue latérale. 
PLANCHE II (suite)

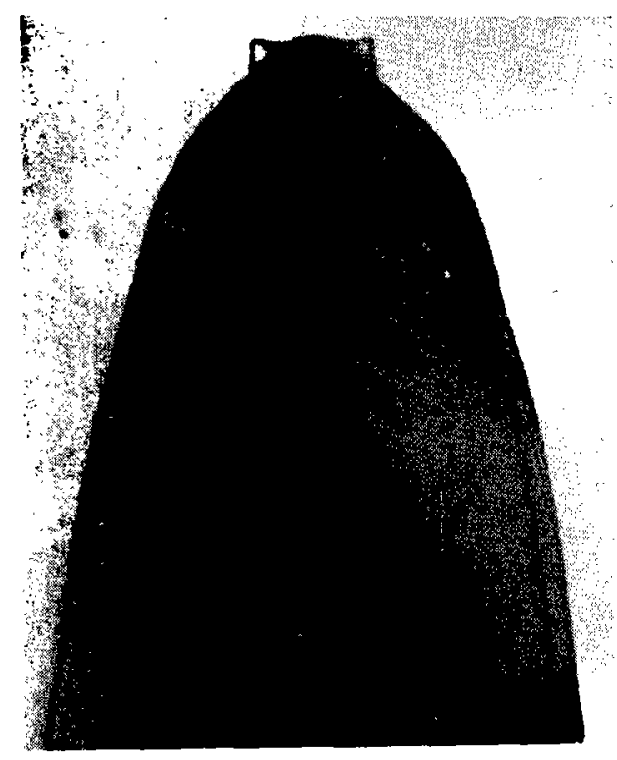

Fo

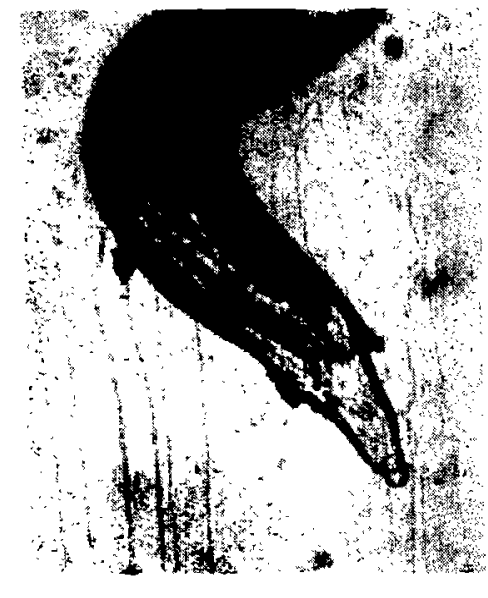

$\mathrm{Fb}$

Setaria digitata : environ $86 \mathrm{~mm}$ de longueur. Cette espèce a un anneau chitineux relativement étroit (en vue latérale) et ce point sera probablement regardé comme une différence typique entre $\mathbf{S}$. labiatopapillosa et $\mathrm{S}$. digitata. (Fa : extrémité anterieure - $\mathrm{Fb}$ : extrémité postérieure).

\section{PLANCHE III}

Dessin à a chambre claire des trois.échantiltons de la filaire oculaire des bœuts irançais. (Comparez avec les photographies $A a$ et $A b$, planche I).
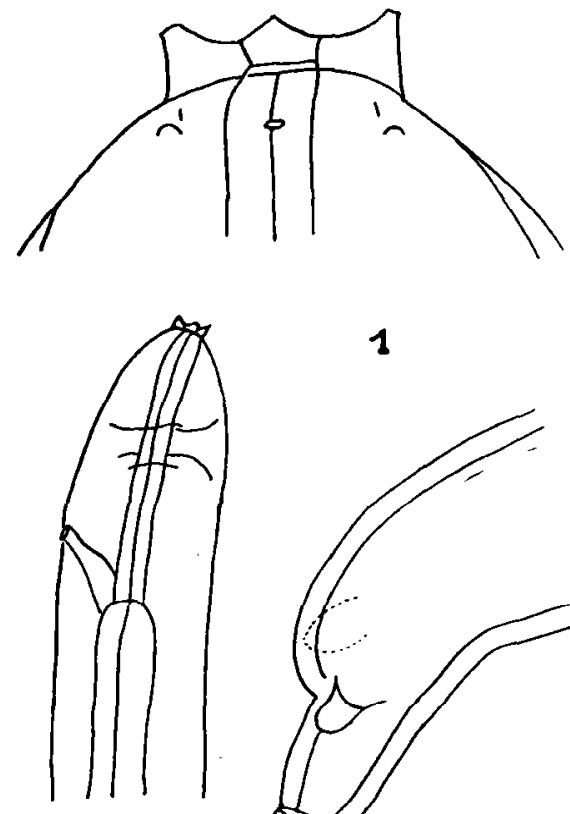

1

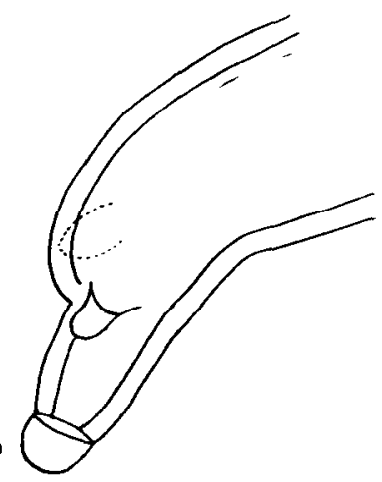

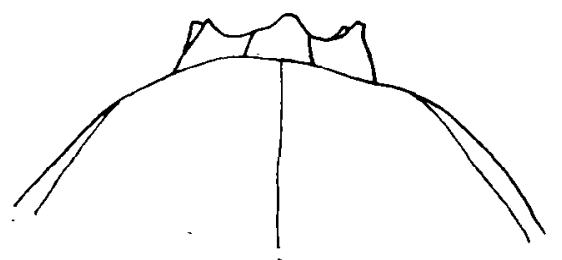

2

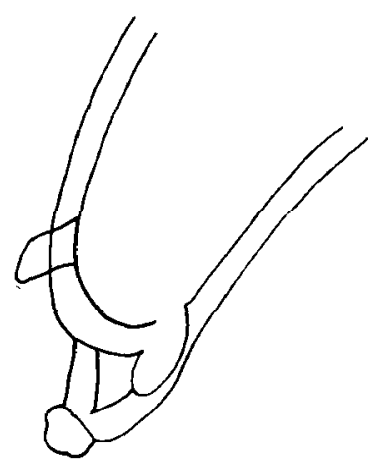

$0,1 \mathrm{~mm}$

3

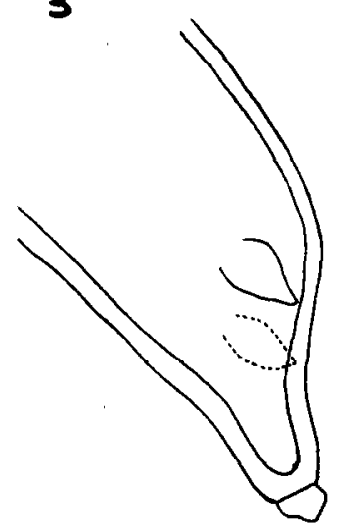


PLANCHE IV

Dessins de filaires oculaires du cheval au Japon.

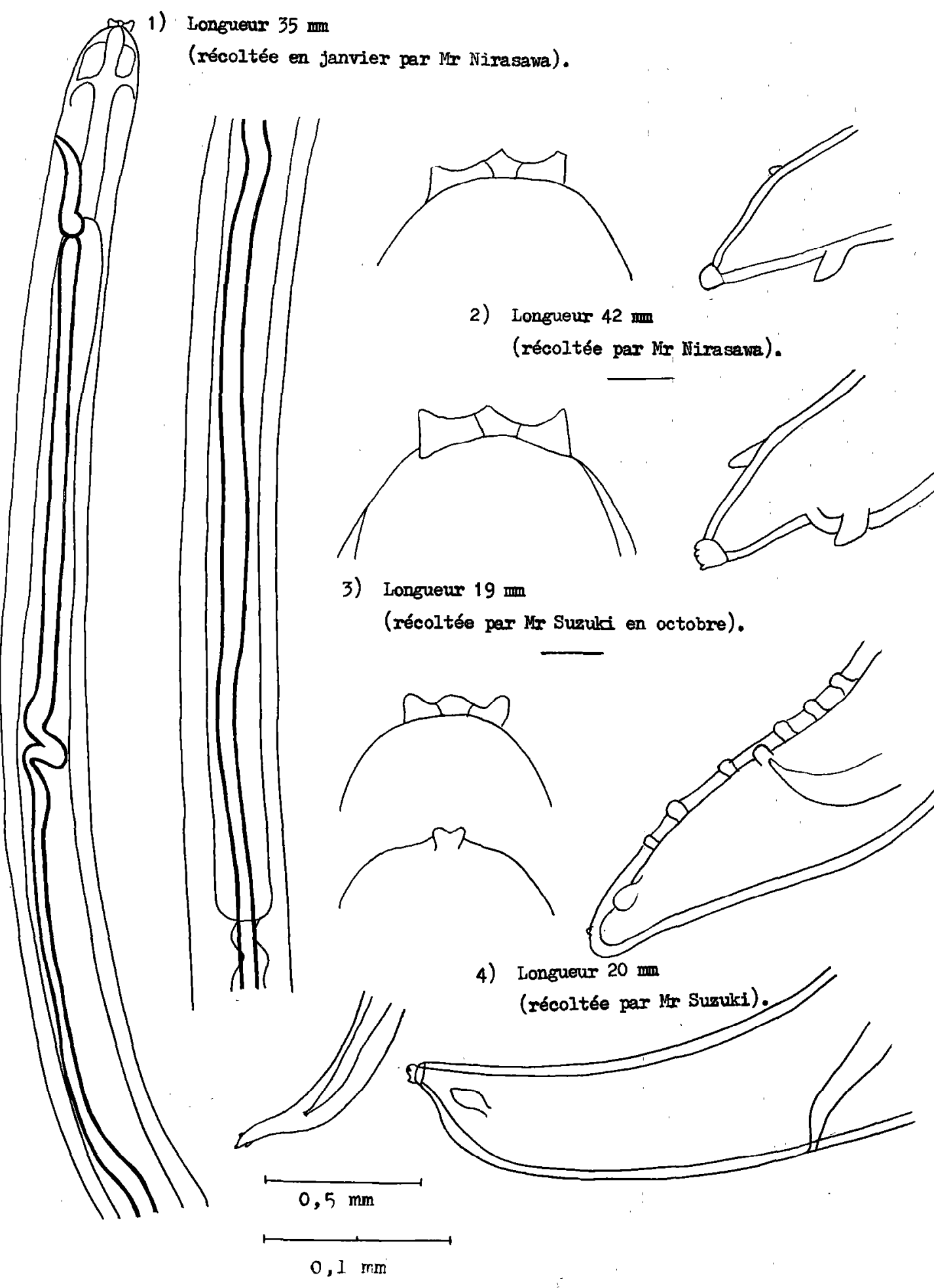




\section{PLANCHE $V$}

Dessins de sétaires bovines adultes (Eemeles) de France, Espagne et Cey'ar.

1) Française, Iongueur $85 \mathrm{~mm}$.
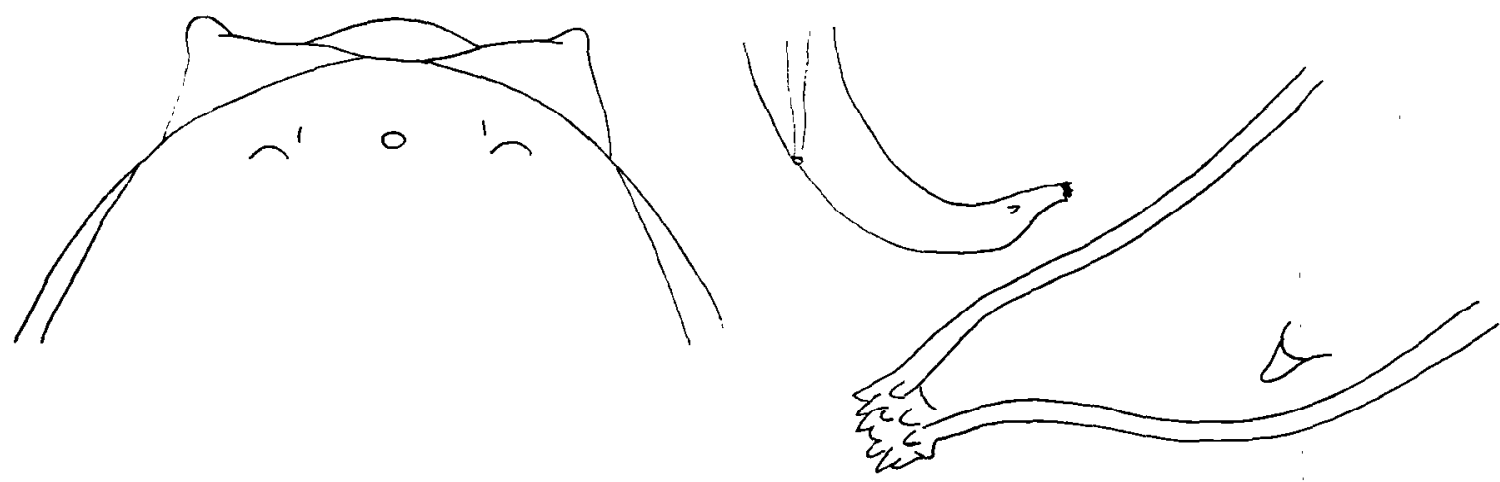

2) Espagnole (Andalousie), longueur $86 \mathrm{~mm}$.
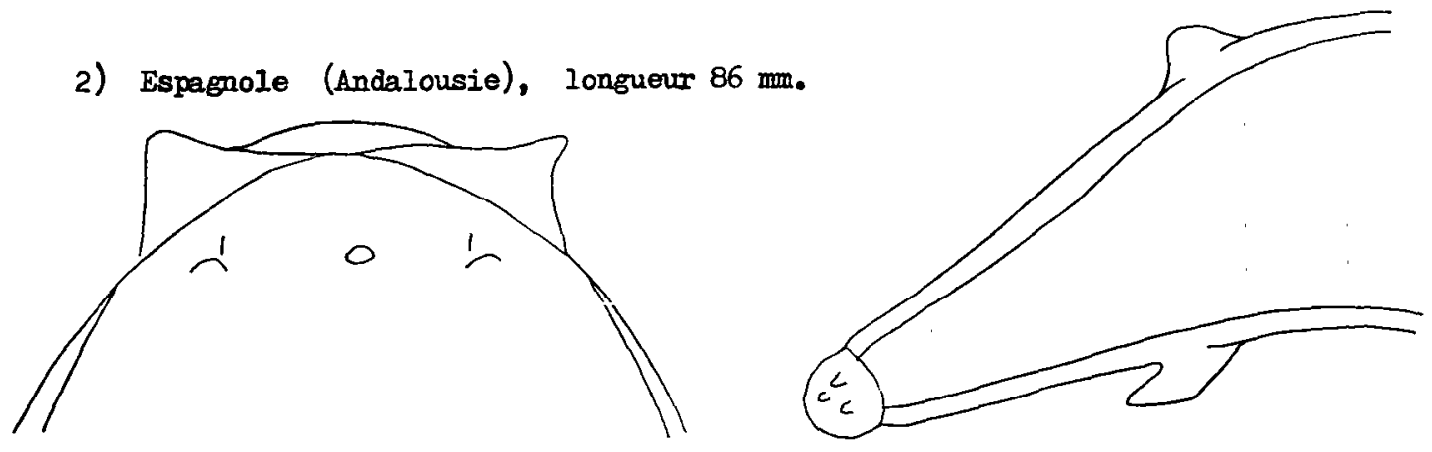

3) Ceylonaise, Iongueur $94 \mathrm{~mm}$.
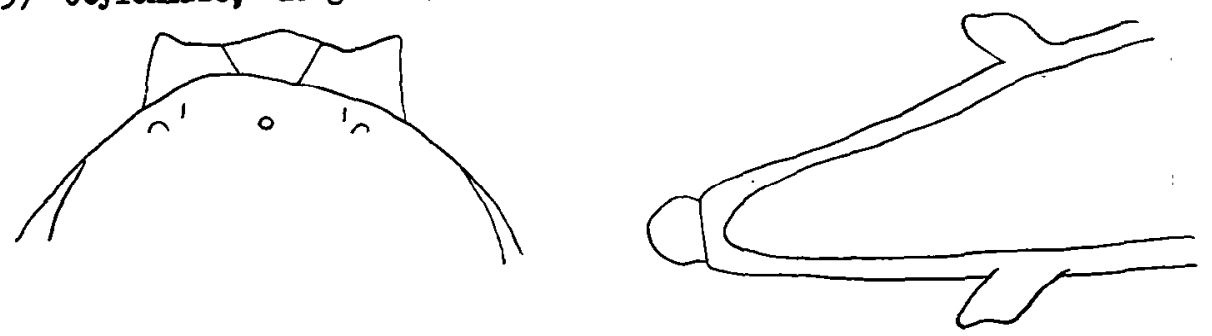

4) Japonaise, longueur $86 \mathrm{~mm}$.
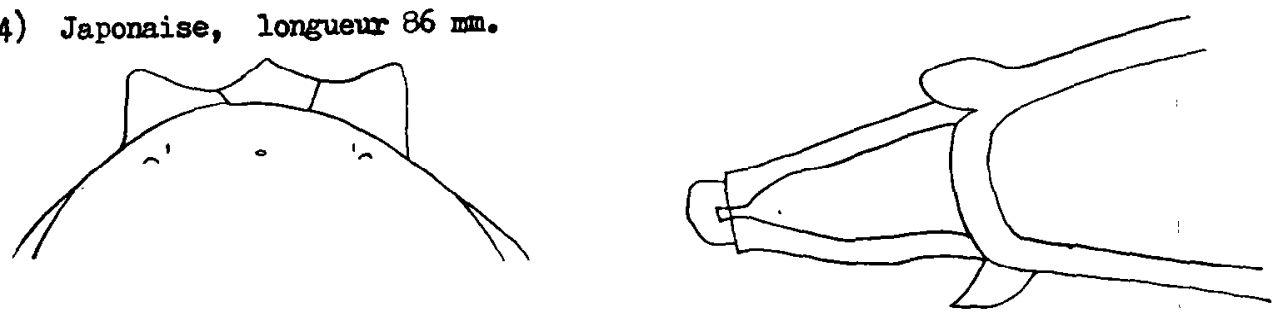

$0,1 \mathrm{~mm}$ 
dices latéraux sub-terminaux est celle qu'on observe habituellement chez les sétaires bovines. Tuutes ces observations m'amènent à la conclusion que les filaires oculaires des boufs français appartiennent à l'espèce S. digitata. Mais une question n'est pas encore résolue, c'est celle de la forme véritable de Setaria labiatopapillosa jeune. Si l'on ne peut pas trouver des individus du type $S$. digitata parmi les sétaires adultes parasites du bœuf en Europe, il est possible que les formes jeunes de S. labiatopapillosa prennent la forme de S. digitata et le phénomène opposé n'est pas impossible froir mon tirage d'un échantillon, recueilli au Japon). Ceł avis n'est pas conforme à celui soutenu par quelques auteurs, qui admettent la différence de S. digitata et S. labiato-papillosa. Actuellement, j'ai seulement cinq échantillons de sétaires adultes provenant de bceufs européens 12 de l'Espagne et 3 de France), qui sont tous S. labiato-papillosa. A mon avis, on peut trouver à la fois S. digitata et S. labiato-papillosa dans les diverses parties du monde. En Asie, où $S$. digitata est généralement prédominante, la région de la péninsule malaise m'a fourni l'association de $S$. digitata et $S$. labiatopapillosa, tandis qu'au Japon et à Ceylan, ou aux Indes du sud je n'ai pas trouvé celle-ci. Quelques chercheurs japonais ont déjà signalé la présence de S. labiato-papillosa au Japon (Yamaguchi, 1943; Yashi, Sato ef Sasa, 1953), mais je ne l'ai pas encore rencontrée dans mes examens qui ont porté sur plus de 300 sétaires adultes, provenant de boeufs japonais indigènes.

A Madras, aux Indes du Sud, Anantaraman et Victor (1957) n'ont trouvé que $S$. digitata chez Bos indicus et Bos bubali, ainsi que je l'ai constaté sur les échantillons envoyés de Madras et d'Orissa, tandis qu'un autre auteur travaillant aux Indes (Sarwar, 1946) a publié une photographie typique de S. labiato-papillosa provenant de sa collection /l'origine de cette sétaire n'est pas mentionnée dans sa publication). Actuellement, le problème de l'identité des deux sétaires bovines faif encore l'objet de mes recherches.

Pour faciliter la comparaison de nos sétaires oculaires avec celles d'Europe où d'autres par. ties du monde, je joins à cette publication des microphotographies de quelques vers recueillis au Japon. Il est intéressant de noter que les parasites des bovidés en ce pays sont trouvés le plus souvent enveloppés d'un tissu réactionnel, formant une légère tumeur sur la paupière supérieure plutôt que nageant, libres, dans la chambre antérieure de l'œil, tandis qu'il n'en est rien chez les équidés. De plus, chez les bovidés, ce phénomène est toujours observé à la saison où on observe le parasitisme chez les équidés. D'autre part, i'ai étudié un cas do sétariose chez un bœuf indigène, chez lequel une tumeur apparut sur une paupière, après évolution de l'affection nerveuse. Le problème complexe qui reste encore posé sur l'identité des sétaires des bovins et des cervidés doit être résolu par l'étude de différences très subtiles, qu'il est très difficile de décrire exactement de façon précise. Pour terminer, je veux ajouter les observations récentes que j'ai faites à Ceylan sur le traitement curatif de l'affection des chevaux, causée par Filaria oculi, avec le Caricide*. Le médicament administré à 2 reprises dans un cas et une seule fois dans un autre cas, à la dose de $80 \mathrm{mg}$ par $\mathrm{kg}$, provoque la disparition du ver nageant dans la chambre antérieure de l'œil' et entraîne l'effacement d'une légère opalescence de l'humeur aqueuse observée avant le traitement. Ce produit chimique nous semble capable de pénétrer très aisément dans le globe oculaire (Shoho, Fernando et Cumarasamy, 1958).

\section{Remerciements :}

J'adresse mes sentiments reconnaissants à M. le Professeur J. Euzéby pour sa collaboration et l'intérêt incessant qu'il a porté à mes recherches. Je remercie également, pour leur arnicale collaboration, mes deux collègues, MM. Nirasawa et Suzuki de la préfecture de Kukushima, qui m'ont fourni leurs récoltes de filaires oculaires nécessaires à mes études.

\section{BIBLIOGRAPHIE}

ABILDGAARD, P.B. (1789). - Zoologia Danica, seu animalium Daniae ef Norvegiae sasiorum ac notorum descriptiones et historiae, 3,49 .

$\left(^{*}\right)$ Produit de $\ll$ Lederle Laboratories, the American Cyanamid Co., New-York ». Présenté par M. le Dr R.L. Burkart. (= Hetrazan = Notézine). II s'agit de la diéthyl-carbamyl-méthyl-pipérazine. 
ANANTARAMAN, M., et VICTOR, D.A. (1957) - Cerebrospinal nematodiasis. I. Setaria of bovines in India. Ind. vet. J., 34, 165.

BAYLIS, H.A. (1936). - On the nomenclature and synonymy of the nematode "Setario labiato-papillosa ». Ann. trop. Med and Parasit., 30, 293.

BÖHM, L.K., und Supperer, R. (1955). - Untersuchungen über Setarien (Nematoda) bei einheimischen Wiederkäuern und deren Beziehungen zur « Epizootischen Cerebrospinalen Nematodiasis " (Setariosis). Z.f. Parasitenkunde, 17, 165.

BAUDET, E.A.R.F., et VERWEY, J.H.P. (1951). - Protostrongyloides cervi n.g. n.sp. als oorzaak van een dodelike bloeding in de schedelholte bij een hert (Cervus elaphus). Tijdschrift v. Diergeneesk., 76, 485.

COBBOLD, T.S. (1879). - Parasites. London: J. \& A. Churchill.

DAVAINE, C. (1877). - Traité des entozoaires et des maladies vermineuses de l'homme ef des animaux domestiques. Paris, J.B. Baillière et fils.

HAYASHI, S., SATO, K., et SASA, M. (1953). - Etude sur les sétaires bovines. J. vet. Med., 116, 43 (Japonais).

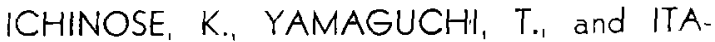
GAKI, S. (1945). - Sur la Filaria oculi. Sogo Juigaku., 11, 69.

INNES, J.R.M., and SAUDERS, L.Z. (1957). Diseases of the central nervous system of domesticatod animals and comparison with human neuropathology. Advances in vet. Sci., 3, 118 ; New-York, acad. Press.

INNES, J.R.M., and SHOHO, C. (1952). Nematodes. Nervous Disease, and neurotro. pic virus infection. Brit. Med. J., Aug. 16, 2. 366.

INNES, J.R.M., and SHOHO, C. (1952). Note sur une nématodose épizootique cérébro-spinale des animaux, forme d'encéphalomyélomalacie focale, causée par de jeunes vers (Sefaria digitata). Acta Neurol., et Psy. Belgica, 7, 417.
KERSCHAGL, W. (1944-45). - Cité par Böhm und Supperer.

LINSIOW, O. von (1906). - Helminths from the collection of the Colombo Museum. Spolia zeylanica, 3, 163.

MAPLESTONE, P.A. (1931). - Parasitic nematodes obtained from animals dying in the Calcutta Zoological Gardens. Parts 4-8. Rec, Ind. Museum (J. Ind. Zoology), 13, 92.

NICHOLLS, L., and CRAWFORD, M. (1925). - Verminous ophthalmia. Ceyl. J. Sci., I, 147.

PERRONCITO, D.E. (1882). - I parasiti dell uomo e degli animali utili. Milano; D.F. Vallardi.

RAILLIET, A.; et HENRY, A. (1911). - Sur une filaire péritonéale des porcins. Bull. Soc. Path. exot, 4, 386.

SCHWANGART, Fr. (1918). - Ursachen der endemischen Parese des Rotwildes. Deutsche Jägerz., 71, 132.

SCHWANGART, Fr. (1940). - Uber die endemischen Parese des Rotwildes und Tuberkulose beim Reh. Berliner. u. münchener tierärtzl. Wochens., 6, 61.

SCHWARTZ, B. (1927). - Nematodes belonging to the Genus Setaria parasitic in the eyes of horses. North Am. Vet., 8, 24.

$\mathrm{SHOHO}, \mathrm{C}$. (1954). - Epizootische cerebrospinale Nematodiasis (Setariasis) und ihre verzweigten Probleme. Deutsche tierärztl. Wochens., 61, 25.

SHOHO, C. (1956). - Cerebro-spinal nematodiasis (Setariasis) in abnormal or inadequate hosts. Ceyl. vet. J., 4, 75.

SHOHO, C. (1958). - Die Setarien aus dem schweizerischen Reh, Capreolus capreolus. Pour Schw. Arch. f. Tierheilk., à paraître. SHOHO, C., FERNANDO, F.D., and CUMARASAMY, K. (1958). - Trials of therapeutic treatment of eye-worm (Filaria oculi) of horses by I-Diethylcarbamyl-4-Methylpiperazine Dihydrogen Citrate. A paraitre.

YAMAGUCHI, S. (1943). - Studies of helminths fauna of Japan. Part 43. Mammalian nematodes, IV. Jap. J. Zoology, 9, 449. 


\section{SUMMARY}

\section{The filaria of bovines and equines}

The author has examined the question whether the filaria of domsstic animals, Setaria labiatopapillosa and $S$. digitata are in fact separate species. He refers to the observations of other parasito'ogists, on ocular and nervous tissue filariasis.

In Korea, it has been established that a cerebro-spinal disorder is due to the immature forms of $S$. digitata. In Japan, a distinction is made between the ocular and nervous types of the disease in the horse particularly. However from observations on certain cases of the oculo-nervous complex, the author thinks that invasion of the eye is through the optical fissure from the brain cavity. The majority of immature filaria found in the eyes of horses in Japan appear to be" S. digi. tata of bovine origin; very rarely are they $S$. equina. The author has examined the filaria in the eyes of bovine in Europe and concludes that these also are S. digitata and he asks accordingly whether, since adult forms of S. digitata of bovines are not found in Europe, it is possible that the young forms of $S$. labiato-papillosa may not take the form of $S$. digitata and vice-versa. He is of the opinion that it is possible to find both species of Setaria in most parts of the world. In Asia S. digitata predominates. His studies continue.

\section{RESUMEN}

\section{Sobre las filarias en los équidos y los bóvidos}

El autor investiga si no hay identidad entre las filarias de los animales domésticos, Setaria labiato-papillosa y S. digitata; recuerda las observaciones de otros parasitólogos concernientes a las filarosis oculares y nerviosas y expone algunas de sus investigaciones. En Corea, se ha establecido que la causa de una enfermedad del sistema nervioso central de los animales domésticos era debida a formas jóvenes de Setaria digitata. En el Japón, se observan diferencias entre las formas de tipo nervioso y las formas de tipo ocular, sobre todo en el caballo. Sin embargo en algunos casos de complejo neuro-ocular observados, el autor piensa que las filarias penetran en el ojo viniendo de la cavidad cerebral por la hendidura óptica. La mayoría de las filarias jóvenes encontradas en el ojo de los caballos del Japon parecen pertenecer a $S$. digitata de origen bovino; las formas de $S$. equina son muy raras.

El autor examinando filarias oculares bovinas de Europa concluye que estas pertenecen tambien a S. digitata; y se pregunta sí, puesto que no se puede encontrar en Europa S. digitata adultas en los bóvidos, no es posible que las formas jóvenes de S. labiato-papillosa tomen la forma de S. digitata y S. labiato-papillosa en diversas partes del mundo; en Asia S. digitata predomina generalmente. El autor continua a estudiar el problema de su identidad. 